\title{
WEAK SOLUTIONS FOR A HYPERBOLIC SYSTEM WITH UNILATERAL CONSTRAINT AND MASS LOSS
}

\author{
SOLUTIONS FAIBLES POUR UN SYTÈME \\ HYPERBOLIQUE AVEC CONTRAINTE UNILATÉRALE \\ ET PERTE DE MASSE
}

\author{
F. BERTHELIN ${ }^{\mathrm{a}}$, F. BOUCHUT ${ }^{\mathrm{b}, *}$ \\ a Université d'Orléans, UMR 6628, département de mathématiques, BP 6759, \\ 45067 Orléans cedex 2, France \\ ${ }^{\mathrm{b}}$ Département de mathématiques et applications, École normale supérieure et CNRS, UMR 8553, \\ 45, rue d'Ulm, 75230 Paris cedex 05, France
}

Received 4 February 2002, accepted 4 November 2002

\begin{abstract}
We consider isentropic gas dynamics equations with unilateral constraint on the density and mass loss. The $\gamma$ and pressureless pressure laws are considered. We propose an entropy weak formulation of the system that incorporates the constraint and Lagrange multiplier, for which we prove weak stability and existence of solutions. The nonzero pressure model is approximated by a kinetic BGK relaxation model, while the pressureless model is approximated by a sticky-blocks dynamics with mass loss.
\end{abstract}

(C) 2003 L'Association Publications de l'Institut Henri Poincaré. Published by Elsevier B.V. All rights reserved

MSC: 76T10; 35L65; 35L85; 76N15; 35A35

Keywords: Conservation laws with constraint; Mass loss; Entropy weak product; Pressureless gas; Sticky blocks

RÉSUMÉ. - Nous considérons les équations de la dynamique des gaz isentropique avec contrainte unilatérale sur la densité et perte de masse. Les lois de pression $\gamma$ et sans pression sont considérées. Nous proposons une formulation faible entropique du système qui incorpore la contrainte et le multiplicateur de Lagrange, pour laquelle nous montrons la stabilité faible et l'existence de solutions. Le modèle avec pression non nulle est approché par un modèle de relaxation BGK cinétique, tandis que le modèle sans pression est approché par une dynamique de bouchons collants avec perte de masse.

(C) 2003 L'Association Publications de l'Institut Henri Poincaré. Published by Elsevier B.V. All rights reserved

\footnotetext{
* Corresponding author.

E-mail addresses: florent.berthelin@ labomath.univ-orleans.fr (F. Berthelin), francois.bouchut@ens.fr (F. Bouchut).
} 


\section{Introduction and models}

\subsection{Models}

The aim of this paper is to introduce a weak formulation and establish weak stability and existence for solutions to some one-dimensional systems of conservations laws with unilateral constraint. Such system arises for example in the modeling of two-phase flows, see [8], as

$$
\left\{\begin{array}{l}
\partial_{t} \rho+\partial_{x}(\rho u)=0, \\
\partial_{t}(\rho u)+\partial_{x}\left(\rho u^{2}+p(\rho)+\pi\right)=0,
\end{array}\right.
$$

with constraint and pressure Lagrange multiplier

$$
0 \leqslant \rho \leqslant 1, \quad \pi \geqslant 0
$$

and extremality relation

$$
(1-\rho) \pi=0 .
$$

This system was studied in [20] with viscosity. Existence and weak stability of suitable weak solutions is obtained in [2] in the pressureless case $p(\rho)=0$, but however, the general nonzero pressure case remains open. We refer to [1,2,16,18-20,22] for other hyperbolic problems with constraints. Some general formulations can be found in [13].

Here we are going to consider a slightly different model with mass loss, that can be written as

$$
\left\{\begin{array}{l}
\partial_{t} \rho+\partial_{x}(\rho u)=Q, \\
\partial_{t}(\rho u)+\partial_{x}\left(\rho u^{2}+p(\rho)\right)=Q u,
\end{array}\right.
$$

with constraint and mass loss rate Lagrange multiplier

$$
0 \leqslant \rho \leqslant 1, \quad Q \leqslant 0,
$$

and extremality relation

$$
(1-\rho) Q=0 .
$$

This model is based on the physical idea to remove from the density $\rho$ what overflows with 1 as rain could make overflow a reservoir or a river. The term $Q u$ on the righthand side of the momentum equation expresses that the overflowing matter travels at velocity $u$. This interpretation is especially relevant for the Saint-Venant equations when $p(\rho)=\kappa \rho^{2}$. We are going to consider here pressure laws of the form

$$
p(\rho)=\kappa \rho^{\gamma}, \quad 1<\gamma \leqslant 3, \kappa \geqslant 0 .
$$

The pressureless case $\kappa=0$ is very particular. Existence and properties for the system of pressureless gas without constraint have been studied in $[9,10,12,15,17]$.

The main difficulty in the model is to give a suitable sense to the products $Q u$ in (1.4) and $\rho Q$ in (1.6), because $Q$ is only a measure, and $\rho, u$ can be discontinuous. Indeed, 
since $Q$ can be nonzero only where $\rho=1$, we have formal formulas obtained by freezing $\rho=1$ in (1.4),

$$
Q=\mathbb{1}_{\rho=1} \partial_{x} u, \quad Q u=\mathbb{1}_{\rho=1}\left(\partial_{t} u+\partial_{x} u^{2}\right),
$$

but of course this is again meaningless. Therefore, we provide the following entropy weak formulation of the problem, that involves what we call entropy weak products. The idea is to introduce a different velocity $v(t, x)$ for the lost matter, and write the system

$$
\left\{\begin{array}{l}
\partial_{t} \rho+\partial_{x}(\rho u)=Q, \\
\partial_{t}(\rho u)+\partial_{x}\left(\rho u^{2}+p(\rho)\right)=Q v
\end{array}\right.
$$

with as before

$$
0 \leqslant \rho \leqslant 1, \quad Q \leqslant 0 .
$$

We take $v \in L^{\infty}(Q)$, so that the product $Q v$ is well-defined as a measure. We need then to formulate in a weak sense that $Q v=Q u$, and that $Q \rho=Q$. In order to do so, we require the family of entropy weak product inequalities

$$
\partial_{t} \eta_{S}(\rho, u)+\partial_{x} G_{S}(\rho, u) \leqslant Q \eta_{S}^{\prime}(1, v) \cdot(1, v)
$$

for any convex entropy $\eta_{S}$ in a suitable family parametrized by a convex function $S$, where $G_{S}$ is its entropy flux, and $\eta_{S}^{\prime}$ is its derivative with respect to $(\rho, \rho u)$. Since $v$, by definition, is defined $Q$ a.e., the term on the right-hand side of (1.11) is well-defined. In order to see that (1.9)-(1.11) is a weak formulation of (1.4)-(1.6), we observe first that any suitable solution to (1.4)-(1.6) also solves (1.9)-(1.11) with $v=u$. Conversely, if we have a sufficiently smooth solution to (1.9)-(1.11), then multiplying (1.9) by $\eta_{S}^{\prime}(\rho, u)$ and comparing with (1.11), we get

$$
Q \eta_{S}^{\prime}(\rho, u) \cdot(1, v) \leqslant Q \eta_{S}^{\prime}(1, v) \cdot(1, v) .
$$

If we take for the entropy the physical energy

$$
\eta(\rho, u)=\rho u^{2} / 2+\frac{\kappa}{\gamma-1} \rho^{\gamma},
$$

we have $\eta^{\prime}(\rho, u)=\left(\gamma \kappa \rho^{\gamma-1} /(\gamma-1)-u^{2} / 2, u\right)$, and (1.12) gives

$$
Q\left[\frac{\gamma \kappa}{\gamma-1}\left(\rho^{\gamma-1}-1\right)-(v-u)^{2} / 2\right] \leqslant 0 .
$$

Together with the constraints (1.10), we deduce that $Q v=Q u$ and $Q \rho=Q$, except in the pressureless case $\kappa=0$, in which we can only conclude $Q v=Q u$. We shall see in Section 4.4 that in this case the formulation really fails to give $Q \rho=Q$ in the strong sense.

Our main result is that the entropy formulation of the system (1.9)-(1.11) is weakly stable. We are able to prove a priori estimates and compactness of suitable approximations, that lead to existence for the Cauchy problem. For the nonzero pressure 
model, the approximate solutions are obtained by a kinetic BGK equation with additional projection to enforce the constraint $\rho \leqslant 1$. For the pressureless model, the approximation is based on the notion of sticky blocks that has been introduced in [8] and used in [2], but here with a different dynamics based on mass loss.

We look for solutions with regularities

$$
\begin{gathered}
\rho \in L_{t}^{\infty}\left(0, \infty ; L_{x}^{\infty}(\mathbb{R}) \cap L_{x}^{1}(\mathbb{R})\right), \\
u \in L_{t}^{\infty}\left(0, \infty ; L_{x}^{\infty}(\mathbb{R})\right), \\
Q \in \mathcal{M}\left(\left[0, \infty[\times \mathbb{R}), \quad v \in L^{\infty}(Q) .\right.\right.
\end{gathered}
$$

The density $\rho$ and the momentum density $\rho u$ are a priori not continuous with respect to time, because $Q$ could contain Dirac distributions in time. However, (1.8) suggests that it should not be the case, but it is an open question to decide whether or not it is the case.

Thus, we consider weak solutions in the sense that for all $\varphi \in \mathcal{D}([0, \infty[\times \mathbb{R})$,

$$
\begin{gathered}
\int_{0}^{\infty} \int_{\mathbb{R}}\left[\rho \partial_{t} \varphi+\rho u \partial_{x} \varphi\right] \mathrm{d} t \mathrm{~d} x+\int_{\mathbb{R}} \rho^{0}(x) \varphi(0, x) \mathrm{d} x=-\int_{[0, \infty[} \int_{\mathbb{R}} \varphi Q \\
\int_{0}^{\infty} \int_{\mathbb{R}}\left[\rho u \partial_{t} \varphi+\left(\rho u^{2}+p(\rho)\right) \partial_{x} \varphi\right] \mathrm{d} t \mathrm{~d} x \\
+\int_{\mathbb{R}} \rho^{0}(x) u^{0}(x) \varphi(0, x) \mathrm{d} x=-\int_{[0, \infty[} \int_{\mathbb{R}} \varphi Q v .
\end{gathered}
$$

It includes the initial data

$$
\rho(0, x)=\rho^{0}(x), \quad \rho(0, x) u(0, x)=\rho^{0}(x) u^{0}(x) .
$$

We assume that $\rho^{0} \in L^{1}(\mathbb{R})$, so that we can bound a priori the mass loss, $\iint-Q \mathrm{~d} t \mathrm{~d} x \leqslant$ $\int \rho^{0} \mathrm{~d} x$.

\subsection{Main results}

The following compactness result is valid for the two possible pressure laws (isentropic model with nonzero pressure or pressureless model).

THEOREM 1.1. - Let us consider a sequence of solutions $\left(\rho_{n}, u_{n}, Q_{n}, v_{n}\right)$ with regularities (1.15)-(1.17) with uniform bounds in their respective spaces of (1.15)(1.17), satisfying (1.18)-(1.19) and (1.10)-(1.11). Initial data $\rho_{n}^{0}, u_{n}^{0}$ are supposed to satisfy

$$
\begin{gathered}
0 \leqslant \rho_{n}^{0} \leqslant 1, \quad\left(\rho_{n}^{0}\right)_{n \geqslant 0} \text { is bounded in } L^{1}(\mathbb{R}), \\
\left(u_{n}^{0}\right)_{n \geqslant 0} \text { is bounded in } L^{\infty}(\mathbb{R}) .
\end{gathered}
$$

In the pressureless case, we also assume that the Oleinik inequality (1.26) holds, and that $Q_{n}$ is bounded in $L_{\mathrm{loc}}^{\infty}(] 0, \infty\left[, \mathcal{M}_{\mathrm{loc}}(\mathbb{R})\right)$. Then, up to a subsequence, as $n \rightarrow \infty$, $\left(\rho_{n}, u_{n}, Q_{n}, v_{n}\right) \rightarrow(\rho, u, Q, v)$ satisfying (1.15)-(1.17), in the following sense 


$$
\begin{gathered}
\rho_{n} \rightarrow \rho, \quad u_{n} \rightarrow u \quad \text { in } L_{w *}^{\infty}(] 0, \infty[\times \mathbb{R}), \\
Q_{n} \rightarrow Q, \quad Q_{n} v_{n} \rightarrow Q v \quad \text { in } \mathcal{M}([0, \infty[\times \mathbb{R}) w *,
\end{gathered}
$$

where $(\rho, u, Q, v)$ is a solution to (1.18)-(1.19) and (1.10)-(1.11) with initial data $\rho^{0}$, $u^{0}$ defined by

$$
\rho_{n}^{0} \rightarrow \rho^{0} \quad \text { in } L_{w *}^{\infty}(\mathbb{R}), \quad \text { and } \quad \rho_{n}^{0} u_{n}^{0} \rightarrow \rho^{0} u^{0} \quad \text { in } L_{w *}^{\infty}(\mathbb{R}) .
$$

In the pressureless case, we also get (1.26). In the nonzero pressure case, we have the convergence a.e. $\rho_{n} \rightarrow \rho, \rho_{n} u_{n} \rightarrow \rho u$.

We turn now to existence. The theorem is again the same for both pressure laws.

THEOREM 1.2. - Let $\rho^{0} \in L^{1}(\mathbb{R})$ such that $0 \leqslant \rho^{0} \leqslant 1$ and $u^{0} \in L^{\infty}(\mathbb{R})$. Then there exists $(\rho, u, Q, v)$ with regularities (1.15)-(1.17) satisfying (1.18)-(1.19) and (1.10)(1.11).

In the pressureless context, we have more precise results.

THEOREM 1.3. - In addition, in the pressureless case $\kappa=0$, the solution of Theorem 1.2 satisfies

$$
\begin{gathered}
\partial_{x} u(t, x) \leqslant \frac{1}{t}, \\
T V_{[a, b]}(u(t, .)) \leqslant \frac{2(b-a)}{t}+2\left\|u^{0}\right\|_{L^{\infty}} \quad \forall a<b, \\
\operatorname{essinf} u^{0}(x) \leqslant u(t, x) \leqslant \operatorname{esssup} u^{0}(x), \\
Q \in L_{\mathrm{loc}}^{\infty}(] 0, \infty\left[, \mathcal{M}_{\mathrm{loc}}(\mathbb{R})\right), \\
\rho, \rho u \in C(] 0, \infty\left[, L^{\infty} w *\right), \\
\left.\partial_{t}(\rho S(u))+\partial_{x}(\rho u S(u))=Q_{S} \quad \text { in }\right] 0, \infty[\times \mathbb{R}
\end{gathered}
$$

for every $S \in C(\mathbb{R})$, where $Q_{S} \in \mathcal{M}([0, \infty[\times \mathbb{R})$ satisfies

$$
\left|Q_{S}\right| \leqslant\|S\|_{L^{\infty}}|Q|
$$

The remainder of the paper is organized as follows. In Section 2, we prove the stability of the entropy weak product formulation. In Section 3, we study the non-zero pressure case. We prove the existence of solutions for a BGK model with relaxation of the type of those introduced in [6]. We obtain kinetic entropy inequalities and the existence of kinetic invariant domains, following [4,24]. Using compensated compactness, we prove the convergence, as $\varepsilon \rightarrow 0$, towards the nonzero pressure gas dynamics model with mass loss. Finally, we provide an alternate analysis of the BGK model in the particular case $\gamma=3$ using averaging lemma. In Section 4, we study the pressureless model. We introduce the sticky blocks dynamics, and specify in this case how entropy weak product inequalities arise. Finally, we prove that the strong extremality relation is lost in weak limits for the pressureless model. 


\section{Stability of the entropy weak product}

The weak stability of the formulation (1.11) becomes clear with the two following lemmas.

LEMMA 2.1. - Let $Q_{n}$ be nonpositive measures and $v_{n} \in L^{\infty}\left(Q_{n}\right)$. If $\left(Q_{n}\right)_{n \geqslant 0}$ is a sequence bounded in $\mathcal{M}_{\mathrm{loc}}\left(\left[0, \infty[\times \mathbb{R})\right.\right.$ and $\left(\left\|v_{n}\right\|_{L^{\infty}\left(Q_{n}\right)}\right)_{n \geqslant 0}$ is bounded, then there exists a measure $Q$ and a function $v \in L^{\infty}(Q)$ such that after extraction of a subsequence, $Q_{n} \rightarrow Q, Q_{n} v_{n} \rightarrow Q v$ and $Q_{n} \varphi\left(v_{n}\right) \rightarrow Q^{\varphi} \leqslant Q \varphi(v)$ for any convex function $\varphi$.

Proof. - The measures $Q_{n}$ are bounded in $\mathcal{M}_{\mathrm{loc}}$, thus for a subsequence $Q_{n} \rightarrow Q$ in $\mathcal{M}_{\text {loc }} w *$. By diagonal extraction, there exists a subsequence such that

$$
Q_{n} \varphi\left(v_{n}\right) \rightarrow Q^{\varphi}
$$

for every $\varphi$ continuous. We have $\left|Q_{n} v_{n}\right| \leqslant C\left|Q_{n}\right|$, thus at the limit $\left|Q^{\text {Id }}\right| \leqslant-C Q$ and therefore there exists $v \in L^{\infty}(Q)$ such that $Q^{\mathrm{Id}}=Q v$.

We compare now $Q^{\varphi}$ and $Q \varphi(v)$ for $\varphi$ convex. A convex function $\varphi$ can be written as

$$
\varphi(v)=\sup \{a v+b ; a, b \text { such that } \varphi \geqslant a \operatorname{Id}+b\} .
$$

Let $\varphi$ be a convex function and let $a, b \in \mathbb{R}$ such that $\varphi \geqslant a \operatorname{Id}+b$. The measure $Q_{n}$ is nonpositive thus $Q_{n} \varphi\left(v_{n}\right) \leqslant Q_{n}\left(a v_{n}+b\right)$, which gives at the limit $Q^{\varphi} \leqslant Q(a v+b)$. Since this is true for any $a, b$ such that $\varphi \geqslant a \mathrm{Id}+b$, we conclude that $Q^{\varphi} \leqslant Q \varphi(v)$.

LEMMA 2.2. - The function $v \mapsto \eta_{S}^{\prime}(1, v) \cdot(1, v)$ is convex for $S: \mathbb{R} \rightarrow \mathbb{R}$ convex and $C^{1}$. Furthermore, it is a nonnegative function as soon as $S \geqslant 0$.

Proof. - We have first to specify what are the entropies $\eta_{S}$. We take the so called weak entropies, that are defined as

$$
\eta_{S}(\rho, u)=\int_{\mathbb{R}} \chi(\rho, \xi-u) S(\xi) \mathrm{d} \xi, \quad S \text { convex }
$$

where $\chi$ is defined by (3.8)-(3.9) in the case $\kappa>0$, and by $\chi(\rho, \xi)=\rho \delta(\xi)$ if $\kappa=0$ (in other words $\left.\eta_{S}(\rho, u)=\rho S(u)\right)$. Recalling that prime denotes differentiation with respect to $(\rho, \rho u)$, we can express the desired quantity and get, for $1<\gamma<3$,

$$
\eta_{S}^{\prime}(1, v) \cdot(1, v)=\frac{1-\theta}{J_{\lambda}} \int_{-1}^{1}\left(1-z^{2}\right)^{\lambda-1} S\left(v+a_{\gamma} z\right) \mathrm{d} z
$$

for $\gamma=3$,

$$
\eta_{S}^{\prime}(1, v) \cdot(1, v)=(S(v+\sqrt{3 \kappa})+S(v-\sqrt{3 \kappa})) / 2,
$$

and for $\kappa=0$,

$$
\eta_{S}^{\prime}(1, v) \cdot(1, v)=S(v)
$$


The result follows obviously.

Remark 2.1. - The sign assertion in Lemma 2.2 is helpful because the right-hand side in (1.11) becomes itself nonpositive when $S \geqslant 0$ and we deduce the decrease of $\int \eta_{S} \mathrm{~d} x$.

\section{Isentropic model with nonzero pressure}

In this section, we introduce a kinetic BGK relaxation model that approximates the problem with nonzero pressure $p(\rho)=\kappa \rho^{\gamma}$ with $\kappa>0,1<\gamma \leqslant 3$. We first prove existence of solutions for the BGK model and establish kinetic invariant domains leading to uniform bounds. Then we let the relaxation parameter $\varepsilon$ tend to 0 , and get an entropy solution to (1.9)-(1.11) via compensated compactness. For the special case $\gamma=3$, an alternate proof via averaging lemma is provided.

\subsection{BGK model}

We consider the following kinetic BGK relaxation model, which is obtained from the one of $[3,4]$ by including an overall projection onto the constraint $\rho \leqslant 1$,

$$
\left.\partial_{t} f+\xi \partial_{x} f=\frac{M^{*}[f]-f}{\varepsilon} \text { in }\right] 0, \infty[\times \mathbb{R} \times \mathbb{R}
$$

where $f=f(t, x, \xi)=\left(f_{0}(t, x, \xi), f_{1}(t, x, \xi)\right) \in \mathbb{R}^{2}, t>0, x \in \mathbb{R}, \xi \in \mathbb{R}$

$$
\begin{gathered}
f(t, x, \xi) \in D_{\xi}, \\
\rho(t, x)=\int_{\mathbb{R}} f_{0}(t, x, \xi) \mathrm{d} \xi, \quad \rho(t, x) u(t, x)=\int_{\mathbb{R}} f_{1}(t, x, \xi) \mathrm{d} \xi, \\
M^{*}[f](t, x, \xi)=M^{*}(\rho(t, x), u(t, x), \xi), \\
M^{*}(\rho, u, \xi)=M(\min (1, \rho), u, \xi),
\end{gathered}
$$

and $M$ is the Maxwellian defined by

$$
\begin{gathered}
M(\rho, u, \xi)=(\chi(\rho, \xi-u),((1-\theta) u+\theta \xi) \chi(\rho, \xi-u)), \\
\chi(\rho, \xi)=c_{\gamma, \kappa}\left(a_{\gamma}^{2} \rho^{\gamma-1}-\xi^{2}\right)_{+}^{\lambda}, \\
\theta=\frac{\gamma-1}{2}, \quad \lambda=\frac{1}{\gamma-1}-\frac{1}{2}, \quad c_{\gamma, \kappa}=\frac{a_{\gamma}^{-2 /(\gamma-1)}}{J_{\lambda}}, \\
J_{\lambda}=\int_{-1}^{1}\left(1-z^{2}\right)^{\lambda} d z=\sqrt{\pi} \Gamma(\lambda+1) / \Gamma(\lambda+3 / 2), \quad a_{\gamma}=\frac{2 \sqrt{\gamma \kappa}}{\gamma-1} .
\end{gathered}
$$

We complete (3.1) by initial data

$$
f(0, x, \xi)=f^{0}(x, \xi)
$$


satisfying energy bounds. For $1<\gamma<3$, we take

$$
D_{\xi}=D=\left\{\left(f_{0}, f_{1}\right) \in \mathbb{R}^{2}, f_{0}>0 \text { or } f_{1}=f_{0}=0\right\},
$$

while if $\gamma=3$,

$$
D_{\xi}=\left\{\left(f_{0}, f_{1}\right) \in \mathbb{R}^{2}, f_{1}=\xi f_{0} \text { and } 0 \leqslant f_{0} \leqslant 1 / 2 \sqrt{3 \kappa}\right\},
$$

and the Maxwellian simplifies in

$$
M(\rho, u, \xi)=\frac{1}{2 \sqrt{3 \kappa}} \mathbb{1}_{u-\sqrt{3 \kappa} \rho<\xi<u+\sqrt{3 \kappa} \rho} K(\xi), \quad K(\xi)=(1, \xi) .
$$

In this case a single scalar equation on $f_{0}$ can be written, the second one on $f_{1}$ being proportional to it.

The entropies involved in the inequality (1.11) are the so called weak entropies, defined by

$$
\eta_{S}(\rho, u)=\int_{\mathbb{R}} \chi(\rho, \xi-u) S(\xi) \mathrm{d} \xi, \quad S \text { convex }
$$

and their entropy fluxes are defined by

$$
G_{S}(\rho, u)=\int_{\mathbb{R}}((1-\theta) u+\theta \xi) \chi(\rho, \xi-u) S(\xi) \mathrm{d} \xi .
$$

To each entropy $\eta_{S}$ we can associate a kinetic entropy, in the case $\gamma<3$ they can be defined from a kernel by

$$
H_{S}(f, \xi)=\int_{\mathbb{R}} \Phi(\rho(f, \xi), u(f, \xi), \xi, v) S(v) \mathrm{d} v, \quad \text { for } f \neq 0, \quad H_{S}(0, \xi)=0
$$

where

$$
\begin{aligned}
& u(f, \xi)=\frac{f_{1} / f_{0}-\theta \xi}{1-\theta}, \\
& \rho(f, \xi)=a_{\gamma}^{-2^{\prime}(\gamma-1)}\left(\left(\frac{f_{1} / f_{0}-\xi}{1-\theta}\right)^{2}+\left(f_{0} / c_{\gamma, \kappa}\right)^{1 / \lambda}\right)^{1 /(\gamma-1)}
\end{aligned}
$$

is the inverse relation for $f=M(\rho, u, \xi)$. The kernel $\Phi$ is symmetric in $\xi, v$ and satisfies in particular $\Phi \geqslant 0$ and $\int_{\mathbb{R}}(1, v) \Phi(\rho, u, \xi, v) \mathrm{d} v=M(\rho, u, \xi)$. For more details about this model, we refer to [4].

For $\gamma=3$, the kinetic entropy is simply given for $f=\left(f_{0}, \xi f_{0}\right) \in D_{\xi}$, by

$$
H_{S}(f, \xi)=S(\xi) f_{0}
$$


so that finally for any $1 \leqslant \gamma \leqslant 3$,

$$
\begin{aligned}
& \eta_{S}(\rho, u)=\int_{\mathbb{R}} H_{S}(M(\rho, u, \xi), \xi) \mathrm{d} \xi, \\
& G_{S}(\rho, u)=\int_{\mathbb{R}} \xi H_{S}(M(\rho, u, \xi), \xi) \mathrm{d} \xi .
\end{aligned}
$$

Let us finally introduce the kinetic invariant domains. The macroscopic invariant domains are defined as follows for any $\omega_{\min }<\omega_{\max }$,

$$
\widetilde{D}=\left\{(\rho, u) \in \mathbb{R}^{+} \times \mathbb{R} ; \rho=0 \text { or } \omega_{\min } \leqslant \omega_{1} \leqslant \omega_{2} \leqslant \omega_{\max }\right\},
$$

where the Riemann invariants $\omega_{1}, \omega_{2}$ are given by

$$
\omega_{1}=u-a_{\gamma} \rho^{(\gamma-1) / 2}, \quad \omega_{2}=u+a_{\gamma} \rho^{(\gamma-1) / 2} .
$$

Their corresponding kinetic invariant domains are defined in the case $1<\gamma<3$ by

$$
\widetilde{D}_{\xi}=\left\{f \in D ; f=0 \text { or } \omega_{\min } \leqslant \omega_{1}(f, \xi) \leqslant \omega_{2}(f, \xi) \leqslant \omega_{\max }\right\},
$$

where

$$
\begin{aligned}
& \omega_{1}(f, \xi)=u(f, \xi)-a_{\gamma} \rho(f, \xi)^{(\gamma-1) / 2}, \\
& \omega_{2}(f, \xi)=u(f, \xi)+a_{\gamma} \rho(f, \xi)^{(\gamma-1) / 2},
\end{aligned}
$$

and in the case $\gamma=3$

$$
\widetilde{D}_{\xi}=\left\{f \in D_{\xi} ; 0 \leqslant f_{0} \leqslant \frac{1}{2 \sqrt{3 \kappa}} \mathbb{1}_{\omega_{\min }<\xi<\omega_{\max }}\right\} .
$$

\subsection{Properties of the kinetic entropy}

We recall the value of the moments of $M$,

$$
\int_{\mathbb{R}} M(\rho, u, \xi) \mathrm{d} \xi=(\rho, \rho u), \quad \int_{\mathbb{R}} \xi M(\rho, u, \xi) \mathrm{d} \xi=\left(\rho u, \rho u^{2}+\kappa \rho^{\gamma}\right),
$$

and

$$
\int_{\mathbb{R}} \frac{1}{2} \xi^{2} M_{0}(\rho, u, \xi) \mathrm{d} \xi=\frac{1}{2} \rho u^{2}+\frac{\kappa}{\gamma-1} \rho^{\gamma} \equiv \eta(\rho, u),
$$

for every $\rho \geqslant 0$ and $u \in \mathbb{R}$. It results from the definition of a convex function that

LEMMA 3.1. - For every $S: \mathbb{R} \rightarrow \mathbb{R}$ convex, we have

$$
\begin{aligned}
& \left(\omega_{2}-\omega_{1}\right) S(\xi)+\left(\omega_{1}-\xi\right) S\left(\omega_{2}\right)+\left(\xi-\omega_{2}\right) S\left(\omega_{1}\right) \\
& \leqslant 0 \quad \text { if } \omega_{1} \leqslant \xi \leqslant \omega_{2}, \\
& \geqslant 0 \quad \text { if } \xi \leqslant \omega_{1} \leqslant \omega_{2} \text { or } \omega_{1} \leqslant \omega_{2} \leqslant \xi .
\end{aligned}
$$


As in [3,4], we need a subdifferential inequality in order to prove boundedness of the entropy, namely

PROPOSITION 3.2 (Subdifferential inequality). - If $S: \mathbb{R} \rightarrow \mathbb{R}$ is convex, of class $C^{1}$, then for every $\rho \geqslant 0, u, \xi \in \mathbb{R}$ and $f \in D_{\xi}$, we have

$$
H_{S}(f, \xi) \geqslant H_{S}(M(\rho, u, \xi), \xi)+T_{S}(\rho, u) \cdot(f-M(\rho, u, \xi)),
$$

with

$$
T_{S}(\rho, u)=\frac{1}{J_{\lambda}} \int_{-1}^{1}\left(1-z^{2}\right)^{\lambda}\left(\begin{array}{c}
S\left(u+a_{\gamma} \rho^{\theta} z\right)+\left(\theta a_{\gamma} \rho^{\theta} z-u\right) S^{\prime}\left(u+a_{\gamma} \rho^{\theta} z\right) \\
S^{\prime}\left(u+a_{\gamma} \rho^{\theta} z\right)
\end{array}\right) \mathrm{d} z,
$$

which coincides with $\eta_{S}^{\prime}(\rho, u)$ when $\rho>0$, where prime denotes differentiation with respect to the conservative variables $(\rho, q \equiv \rho u)$. We also have if $f \neq 0$

$$
\left(H_{S}^{\prime}(f, \xi)-T_{S}(\rho, u)\right) \cdot(M(\rho, u, \xi)-f) \leqslant 0,
$$

where if $\gamma=3, H_{S}^{\prime}(f, \xi)=S(\xi)(1,0)$ by convention.

Proof. - The case $\gamma<3$ was treated in [4], thus let us assume that $\gamma=3$. We set $\omega_{1}=u-\sqrt{3 \kappa} \rho, \omega_{2}=u+\sqrt{3 \kappa} \rho$, thus $\omega_{2}-\omega_{1}=2 \sqrt{3 \kappa} \rho \geqslant 0$. We have for $\rho>0$

$$
\begin{aligned}
T_{S}(\rho, u) & =\frac{1}{2 \sqrt{3 \kappa} \rho}\left(\begin{array}{c}
(\sqrt{3 \kappa} \rho-u) S(u+\sqrt{3 \kappa} \rho)+(\sqrt{3 \kappa} \rho+u) S(u-\sqrt{3 \kappa} \rho) \\
S(u+\sqrt{3 \kappa} \rho)-S(u-\sqrt{3 \kappa} \rho)
\end{array}\right) \\
& =\frac{1}{\omega_{2}-\omega_{1}}\left(\begin{array}{c}
-\omega_{1} S\left(\omega_{2}\right)+\omega_{2} S\left(\omega_{1}\right) \\
S\left(\omega_{2}\right)-S\left(\omega_{1}\right)
\end{array}\right),
\end{aligned}
$$

and $T_{S}(0, u)=\left(S(u)-u S^{\prime}(u), S^{\prime}(u)\right)$. We compute

$$
\begin{aligned}
A & =H_{S}(f, \xi)-H_{S}(M(\rho, u, \xi), \xi)-T_{S}(\rho, u) \cdot(f-M(\rho, u, \xi)) \\
& =\left[f_{0}-M_{0}(\rho, u, \xi)\right]\left[S(\xi)-T_{S}(\rho, u) \cdot(1, \xi)\right] .
\end{aligned}
$$

Then, if $\rho>0$,

$$
\begin{aligned}
& S(\xi)-T_{S}(\rho, u) \cdot(1, \xi) \\
& \quad=\left[\left(\omega_{2}-\omega_{1}\right) S(\xi)+\left(\omega_{1}-\xi\right) S\left(\omega_{2}\right)+\left(\xi-\omega_{2}\right) S\left(\omega_{1}\right)\right] /\left(\omega_{2}-\omega_{1}\right),
\end{aligned}
$$

and if $\rho=0$,

$$
S(\xi)-T_{S}(\rho, u) \cdot(1, \xi)=S(\xi)-S(u)-S^{\prime}(u)(\xi-u) \geqslant 0 .
$$

If $\xi \leqslant \omega_{1}$ or $\xi \geqslant \omega_{2}$, then $M_{0}(\rho, u, \xi)=0$, and $S(\xi)-T_{S}(\rho, u) \cdot(1, \xi) \geqslant 0$ thanks to Lemma 3.1, thus $A \geqslant 0$. If $\omega_{1}<\xi<\omega_{2}$, then $M_{0}(\rho, u, \xi)=1 /(2 \sqrt{3 \kappa}) \geqslant f_{0}$ and $S(\xi)-T_{S}(\rho, u) \cdot(1, \xi) \leqslant 0$, thus $A \geqslant 0$ also, which proves (3.26) and (3.28).

The kinetic entropy associated to the physical energy is denoted by $H=H_{v^{2} / 2}$. We recall that $H \geqslant 0$. Applying the previous result to $\min (1, \rho)$ and integrating in $\xi$, we get a minimization principle. 
Corollary 3.3 (Entropy minimization principle). - Assume that $S: \mathbb{R} \rightarrow \mathbb{R}$ is convex of class $C^{1}$ and such that $|S(v)| \leqslant B\left(1+v^{2}\right)$ for some $B \geqslant 0$. Consider $f \in L^{1}\left(\mathbb{R}_{\xi}\right)$ such that $f \in D_{\xi}$ a.e. and

$$
\int_{\mathbb{R}} H(f(\xi), \xi) \mathrm{d} \xi<\infty .
$$

Then $H_{S}(f(\xi), \xi)$ and $H_{S}\left(M^{*}[f](\xi), \xi\right)$ lie in $L^{1}\left(\mathbb{R}_{\xi}\right)$, and setting $(\rho, \rho u)=\int_{\mathbb{R}} f \mathrm{~d} \xi$ we have

$$
\int_{\mathbb{R}} H_{S}\left(M^{*}[f](\xi), \xi\right) \mathrm{d} \xi+T_{S}(\min (1, \rho), u) \cdot(1, u)(\rho-1)_{+} \leqslant \int_{\mathbb{R}} H_{S}(f(\xi), \xi) \mathrm{d} \xi .
$$

For further reference, we also provide the following obvious estimate.

LEMMA 3.4. - For every $\rho \geqslant 0$ and $u, \xi \in \mathbb{R}$, we have

$$
0 \leqslant M_{0}^{*}(\rho, u, \xi) \leqslant M_{0}(\rho, u, \xi) .
$$

\subsection{Existence for the BGK model}

We proceed as in [3] in order to apply Schauder's theorem and to get the existence of global solutions. We notice that the proofs simplify for $\gamma=3$ because $H(f, \xi)=f_{0} \xi^{2} / 2$ is linear and because the kinetic system becomes in fact a rank-one model, and as a consequence we mainly only study the convergence of the first component which is nonnegative. In any case we get the following result.

THEOREM 3.5. - Assume that $f^{0} \in L^{1}\left(\mathbb{R}_{x} \times \mathbb{R}_{\xi}\right)$ satisfies $f^{0}(x, \xi) \in D_{\xi}$ a.e. in $\mathbb{R} \times \mathbb{R}$ and

$$
\iint_{\mathbb{R} \times \mathbb{R}} H\left(f^{0}(x, \xi), \xi\right) \mathrm{d} x \mathrm{~d} \xi=C_{H}^{0}<\infty
$$

Then there exists a solution $f$ to (3.1)-(3.12) satisfying

$$
\begin{gathered}
f \in\left(C \cap L^{\infty}\right)_{t}\left(\left[0, \infty\left[, L^{1}\left(\mathbb{R}_{x} \times \mathbb{R}_{\xi}\right)\right),\right.\right. \\
\forall t \geqslant 0, \quad f(t, x, \xi) \in D_{\xi} \quad \text { a.e. in } \mathbb{R} \times \mathbb{R}, \\
\forall t \geqslant 0, \iint_{\mathbb{R} \times \mathbb{R}} f_{0}(t, x, \xi) \mathrm{d} x \mathrm{~d} \xi \leqslant \iint_{\mathbb{R} \times \mathbb{R}} f_{0}^{0}(x, \xi) \mathrm{d} x \mathrm{~d} \xi, \\
\forall t \geqslant 0, \quad \iint_{\mathbb{R} \times \mathbb{R}} H(f(t, x, \xi), \xi) \mathrm{d} x \mathrm{~d} \xi \leqslant C_{H}^{0}, \\
\partial_{t}\left(\int_{\mathbb{R}} f_{0} \mathrm{~d} \xi\right)+\partial_{x}\left(\int_{\mathbb{R}} \xi f_{0} \mathrm{~d} \xi\right)=-\frac{(\rho-1)_{+}}{\varepsilon} \leqslant 0,
\end{gathered}
$$




$$
\begin{aligned}
\frac{\mathrm{d}}{\mathrm{d} t} \iint_{\mathbb{R} \times \mathbb{R}} H(f(t, x, \xi), \xi) \mathrm{d} x \mathrm{~d} \xi \\
=-\frac{1}{\varepsilon} \iint_{\mathbb{R} \times \mathbb{R}}\left(H^{\prime}(f, \xi)-\left(\frac{\gamma \kappa}{\gamma-1}\left(\rho^{*}\right)^{\gamma-1}-\frac{u^{2}}{2}, u\right)\right) \cdot\left(f-M^{*}[f]\right) \mathrm{d} x \mathrm{~d} \xi \\
\quad-\frac{1}{\varepsilon}\left(\frac{\gamma \kappa}{\gamma-1}\left(\rho^{*}\right)^{\gamma-1}+\frac{u^{2}}{2}\right)(\rho-1)_{+} \leqslant 0,
\end{aligned}
$$

where $(\rho, \rho u)=\int_{\mathbb{R}} f \mathrm{~d} \xi$ and $\rho^{*}=\min (1, \rho)$.

We notice that we have a representation of the solution $f$,

$$
f(t, x, \xi)=f^{0}(x-t \xi, \xi) \mathrm{e}^{-t / \varepsilon}+\frac{1}{\varepsilon} \int_{0}^{t} \mathrm{e}^{-s / \varepsilon} M^{*}[f](t-s, x-s \xi, \xi) \mathrm{d} s .
$$

\subsection{Kinetic invariant domains}

By using Corollary 3.3 and Lemma 2.2 in a computation similar to (3.41), we get

PROPOSITION 3.6. - Assume that $S: \mathbb{R} \rightarrow \mathbb{R}$ is convex of class $C^{1}$ and such that $0 \leqslant S(v) \leqslant B\left(1+v^{2}\right)$ for some $B \geqslant 0$. Then, with $f$ the solution of Theorem 3.5,

$$
\iint_{\mathbb{R} \times \mathbb{R}} H_{S}(f(t, x, \xi), \xi) \mathrm{d} x \mathrm{~d} \xi \leqslant \iint_{\mathbb{R} \times \mathbb{R}} H_{S}\left(f^{0}(x, \xi), \xi\right) \mathrm{d} x \mathrm{~d} \xi .
$$

Noticing that the invariant domain (3.20) is stable by the projection $(\rho, u) \mapsto$ $(\min (1, \rho), u)$, this allows to obtain invariant domains and bounds for $\rho, u, f$ and $M^{*}[f]$.

THEOREM 3.7. - For any $\omega_{\min }<\omega_{\max }$, the system (3.1) has the property that $\widetilde{D}_{\xi}$ is a family of convex kinetic invariant domains. Moreover, the set $\widetilde{D}_{\xi}$ is associated with the invariant domain $\widetilde{D}$ in the sense that

$$
\forall(\rho, u) \in \widetilde{D}, \quad M(\rho, u, \xi) \in \widetilde{D}_{\xi} \text { a.e. } \xi,
$$

and

$$
\begin{aligned}
& \text { for any } f(\xi) \in L^{1}\left(\mathbb{R}_{\xi}\right) \text { such that } f(\xi) \in \widetilde{D}_{\xi} \text { a.e. } \xi \\
& \qquad(\rho, u) \in \widetilde{D} \text { with }(\rho, \rho u)=\int_{\mathbb{R}} f(\xi) \mathrm{d} \xi .
\end{aligned}
$$

In particular, if the initial data of Theorem 3.5 satisfies $f^{0}(x, \xi) \in \widetilde{D}_{\xi}$ for a.e. $x$, $\xi$, then, denoting by $f$ the solution obtained in Theorem 3.5, $(\rho, u)$ defined by (3.3) verify $\forall t \geqslant 0,(\rho(t, x), u(t, x)) \in \widetilde{D}$ for a.e. $x$.

Besides $f(t, x, \xi) \in \widetilde{D}_{\xi} \forall t \geqslant 0$ and consequently $f$ has compact support with respect to $\xi, \operatorname{supp}_{\xi} f \subset\left[\omega_{\min }, \omega_{\max }\right]$.

Furthermore $\rho, u, f, M[f]$ and $M^{*}[f]$ are uniformly bounded in $L^{\infty}$. 
Proof. - Using the functions $S(v)=\left(v-\omega_{\max }\right)_{+}^{2}, S(v)=\left(\omega_{\min }-v\right)_{+}^{2}$ in Proposition 3.6, and the fact that

$$
\left.\mathbb{1}_{(\xi-u)^{2}<3 \kappa \rho^{2}} \leqslant \mathbb{1}_{\omega_{\min }<\xi<\omega_{\max }} \Leftrightarrow\right] u-\sqrt{3 \kappa} \rho, u+\sqrt{3 \kappa} \rho[\subset] \omega_{\min }, \omega_{\max }[,
$$

we obtain the result by adapting the proof of [4].

\subsection{Relaxation limit via compensated compactness}

In this section, we prove the stability Theorem 1.1 and the existence Theorem 1.2 in the case of nonzero pressure.

Proof of Theorem 1.1 for nonzero pressure. - Let $\left(\rho_{n}, u_{n}, Q_{n}, v_{n}\right)$ satisfy the assumptions of Theorem 1.1. Then

$$
\partial_{t} \eta_{S}\left(\rho_{n}, u_{n}\right)+\partial_{x} G_{S}\left(\rho_{n}, u_{n}\right) \leqslant Q_{n} \eta_{S}^{\prime}\left(1, v_{n}\right) \cdot\left(1, v_{n}\right),
$$

and since the right-hand side is bounded in $\mathcal{M}_{\text {loc }}$, we can apply the compensated compactness result of [21] and it gives that, up to a subsequence, $\left(\rho_{n}, \rho_{n} u_{n}\right)$ converge a.e. in $] 0, \infty[\times \mathbb{R}$ when $n \rightarrow \infty$ to some functions $(\rho, \rho u)$. Using Lemmas 2.1 and 2.2, we can pass to the limit in (3.46), while the limit in (1.18)-(1.19) is obvious.

We turn now to the existence result (Theorem 1.2) and prove the relaxation of (3.1) to (1.9)-(1.11).

THEOREM 3.8. - Let us denote by $f_{\varepsilon}$ the solution of Theorem 3.5 with the same initial data $f^{0}(x, \xi) \in L^{1}(\mathbb{R} \times \mathbb{R})$ that satisfies $f^{0}(x, \xi) \in \widetilde{D}_{\xi}$ a.e. for some $\omega_{\min }<\omega_{\max }$, and the energy bound (3.35). Then $\left(\rho_{\varepsilon}, u_{\varepsilon}\right)$ defined by (3.3) are uniformly bounded in $L^{\infty}$, and passing if necessary to subsequences, $\left(\rho_{\varepsilon}, \rho_{\varepsilon} u_{\varepsilon}\right)$ converge a.e. in $] 0, \infty[\times \mathbb{R}$ when $\varepsilon \rightarrow 0$ to $(\rho, \rho u),-\left(\rho_{\varepsilon}-1\right)_{+} / \varepsilon \rightarrow Q,-\left(\rho_{\varepsilon}-1\right)_{+} u_{\varepsilon} / \varepsilon \rightarrow Q v$, where $(\rho, u, Q, v)$ have the regularities (1.15)-(1.17) and satisfy (1.18)-(1.19) and (1.10)-(1.11), with initial data $\left(\rho^{0}, \rho^{0} u^{0}\right)=\int f^{0} \mathrm{~d} \xi$.

Proof. - The bounds of Theorem 3.7 give that $\rho_{\varepsilon}, u_{\varepsilon} f_{\varepsilon}$ and the support in $\xi$ of $f_{\varepsilon}$ are uniformly bounded. Then, the renormalization result for a transport equation of [7] gives for any convex $C^{1}$ function $S$

$$
\partial_{t} H_{S}\left(f_{\varepsilon}, \xi\right)+\xi \partial_{x} H_{S}\left(f_{\varepsilon}, \xi\right)=H_{S}^{\prime}\left(f_{\varepsilon}, \xi\right) \cdot\left(M^{*}\left[f_{\varepsilon}\right]-f_{\varepsilon}\right) / \varepsilon
$$

By integration in $\xi$, it yields

$$
\begin{aligned}
& \partial_{t} \int_{\mathbb{R}} H_{S}\left(f_{\varepsilon}, \xi\right) \mathrm{d} \xi+\partial_{x} \int_{\mathbb{R}} \xi H_{S}\left(f_{\varepsilon}, \xi\right) \mathrm{d} \xi \\
& =\frac{1}{\varepsilon} \int_{\mathbb{R}}\left(H_{S}^{\prime}\left(f_{\varepsilon}, \xi\right)-T_{S}\left(\rho_{\varepsilon}^{*}, u_{\varepsilon}\right)\right) \cdot\left(M^{*}\left[f_{\varepsilon}\right]-f_{\varepsilon}\right) \mathrm{d} \xi \\
& \quad+T_{S}\left(\rho_{\varepsilon}^{*}, u_{\varepsilon}\right) \cdot \int_{\mathbb{R}} \frac{M^{*}\left[f_{\varepsilon}\right]-f_{\varepsilon}}{\varepsilon} \mathrm{d} \xi,
\end{aligned}
$$


with $\rho_{\varepsilon}^{*}=\min \left(1, \rho_{\varepsilon}\right)$. Define

$$
Q_{\varepsilon}=-\frac{\left(\rho_{\varepsilon}-1\right)_{+}}{\varepsilon} \leqslant 0
$$

Then

$$
\int_{\mathbb{R}} \frac{M^{*}\left[f_{\varepsilon}\right]-f_{\varepsilon}}{\varepsilon} \mathrm{d} \xi=Q_{\varepsilon}\left(1, u_{\varepsilon}\right)
$$

and

$$
Q_{\varepsilon} T_{S}\left(\rho_{\varepsilon}^{*}, u_{\varepsilon}\right)=Q_{\varepsilon} T_{S}\left(1, u_{\varepsilon}\right)=Q_{\varepsilon} \eta_{S}^{\prime}\left(1, u_{\varepsilon}\right)
$$

thus by (3.28)

$$
\begin{aligned}
\partial_{t} \eta_{S}\left(\rho_{\varepsilon}^{*}, u_{\varepsilon}\right)+\partial_{x} G_{S}\left(\rho_{\varepsilon}^{*}, u_{\varepsilon}\right) \leqslant & \partial_{t} \int_{\mathbb{R}}\left(H_{S}\left(M^{*}\left[f_{\varepsilon}\right], \xi\right)-H_{S}\left(f_{\varepsilon}, \xi\right)\right) \mathrm{d} \xi \\
& +\partial_{x} \int_{\mathbb{R}} \xi\left(H_{S}\left(M^{*}\left[f_{\varepsilon}\right], \xi\right)-H_{S}\left(f_{\varepsilon}, \xi\right)\right) \mathrm{d} \xi \\
& +Q_{\varepsilon} \eta_{S}^{\prime}\left(1, u_{\varepsilon}\right) \cdot\left(1, u_{\varepsilon}\right) .
\end{aligned}
$$

Next, we observe that $Q_{\varepsilon}$ is bounded in $L^{1}(] 0, \infty[\times \mathbb{R})$ since

$$
\iint-Q_{\varepsilon} \mathrm{d} x \mathrm{~d} t \leqslant \iint_{\mathbb{R} \times \mathbb{R}} f_{0}^{0}(x, \xi) \mathrm{d} x \mathrm{~d} \xi,
$$

as a consequence of (3.40). We deduce that $\left(\rho_{\varepsilon}-1\right)_{+}$tends to 0 in $L^{1}$, and after extraction of a subsequence, $\rho_{\varepsilon}-\rho_{\varepsilon}^{*} \rightarrow 0$ a.e. Provided that $f_{\varepsilon}-M^{*}\left[f_{\varepsilon}\right] \rightarrow 0$ a.e. $t, x, \xi$, using (3.51), we can then apply the compensated compactness result of [21] which gives that up to a subsequence, $\left(\rho_{\varepsilon}^{*}, \rho_{\varepsilon}^{*} u_{\varepsilon}\right)$ (and also $\left(\rho_{\varepsilon}, \rho_{\varepsilon} u_{\varepsilon}\right)$ ) converge a.e. in $] 0, \infty[\times \mathbb{R}$ when $\varepsilon \rightarrow 0$ to some $(\rho, \rho u)$, with $(\rho, u) \in \widetilde{D}, 0 \leqslant \rho \leqslant 1$. By applying Lemma 2.1, we get $Q_{\varepsilon} \rightarrow Q, Q_{\varepsilon} u_{\varepsilon} \rightarrow Q v$ with $Q \in \mathcal{M}\left(\left[0, \infty[\times \mathbb{R}), v \in L^{\infty}(Q)\right.\right.$. Using again $f_{\varepsilon}-M^{*}\left[f_{\varepsilon}\right] \rightarrow 0$ a.e. $t, x, \xi$ and with Lemma 2.2, the limit in (3.51) gives (1.11). A direct integration of (3.1) also gives (1.18)-(1.19) at the limit. Thus it only remains to prove that $f_{\varepsilon}-M^{*}\left[f_{\varepsilon}\right] \rightarrow 0$ a.e. $t, x, \xi$. This can be justified as follows. From (3.41), the integral

$$
\iiint_{] 0, T[\times \mathbb{R} \times \mathbb{R}}\left(H^{\prime}\left(f_{\varepsilon}, \xi\right)-T_{v^{2} / 2}\left(\rho_{\varepsilon}^{*}, u_{\varepsilon}\right)\right) \cdot \frac{M^{*}\left[f_{\varepsilon}\right]-f_{\varepsilon}}{\varepsilon} \mathrm{d} t \mathrm{~d} x \mathrm{~d} \xi
$$

is bounded uniformly in $\varepsilon$. Thus, if $\gamma<3$, we can adapt the dissipation result of [4] and get that $f_{\varepsilon}-M^{*}\left[f_{\varepsilon}\right] \rightarrow 0$ a.e. $t, x, \xi$, replacing the use of the identity $\int_{\mathbb{R}}\left(f_{\varepsilon}\right)_{0} \mathrm{~d} \xi=$ $\int_{\mathbb{R}}\left(M\left[f_{\varepsilon}\right]\right)_{0} \mathrm{~d} \xi$ by

$$
0 \leqslant \iiint_{(t, x) \in B}\left(\left(f_{\varepsilon}\right)_{0}-\left(M^{*}\left[f_{\varepsilon}\right]\right)_{0}\right) \mathrm{d} t \mathrm{~d} x \mathrm{~d} \xi \leqslant K \varepsilon
$$


for any bounded set $B$. For $\gamma=3$, the study is a little bit different and we refer to the next section for the precise analysis, that leads to the same result $f_{\varepsilon}-M^{*}\left[f_{\varepsilon}\right] \rightarrow 0$ a.e. $t, x, \xi$.

Proof of Theorem 1.2. - Let $\rho^{0}, u^{0}$ satisfy $\rho^{0} \in L^{1}(\mathbb{R}), 0 \leqslant \rho^{0} \leqslant 1$ and $u^{0} \in$ $L^{\infty}(\mathbb{R})$. Then there exists $\omega_{\min }, \omega_{\max }$ such that $\left(\rho^{0}, u^{0}\right) \in \widetilde{D}$ a.e. We take $f^{0}(x, \xi)=$ $M\left(\rho^{0}(x), u^{0}(x), \xi\right) \in \widetilde{D}_{\xi}$. Since

$$
\iint_{\mathbb{R} \times \mathbb{R}} H\left(M\left(\rho^{0}, u^{0}, \xi\right), \xi\right) \mathrm{d} x \mathrm{~d} \xi=\int_{\mathbb{R}} \eta\left(\rho^{0}, u^{0}\right) \mathrm{d} x<\infty,
$$

we can apply Theorem 3.8 and we get the result.

\subsection{Relaxation limit for $\gamma=3$ via averaging lemma}

In this section, we complete the proof of Theorem 3.8 in the case $\gamma=3$ by proving that $f_{\varepsilon}-M^{*}\left[f_{\varepsilon}\right] \rightarrow 0$ a.e. $t, x, \xi$ also in this case, and we give an alternate compactness argument via averaging lemma instead of compensated compactness, following the ideas of $[11,14,23,25,26]$. Let $f_{\varepsilon}$ be the solution of Theorem 3.5 with the same initial data $f^{0}(x, \xi)$ and $\left(\rho_{\varepsilon}, u_{\varepsilon}\right)$ be the approximate solutions to (1.9) defined by (3.3). In order to prove the compactness of $\rho_{\varepsilon}$ and $\rho_{\varepsilon} u_{\varepsilon}$, we use the compactness averaging lemma of [14] in the following form.

Proposition 3.9. - Let $g_{\varepsilon} \in L^{\infty}(] 0, \infty[\times \mathbb{R} \times \mathbb{R})$ satisfy

$$
\partial_{t} g_{\varepsilon}+\xi \partial_{x} g_{\varepsilon}=-\lambda_{\varepsilon}-\partial_{\xi \xi}^{2} \mu_{\varepsilon}
$$

for some nonnegative measures $\lambda_{\varepsilon}, \mu_{\varepsilon}$ locally bounded uniformly in $\varepsilon$. If $g_{\varepsilon}$ is bounded in $L^{\infty}$ uniformly in $\varepsilon$, then $\int_{\mathbb{R}} g_{\varepsilon}(t, x, \xi) \psi(\xi) \mathrm{d} \xi$ belongs to a compact set of $L_{\mathrm{loc}}^{p}(] 0, \infty[\times \mathbb{R}), 1<p<\infty$, for any $\psi \in C_{c}^{\infty}(\mathbb{R})$.

PROPOSITION 3.10. - The solution $f_{\varepsilon}$ of Theorem 3.5 for $\gamma=3$ satisfies

$$
\partial_{t}\left(f_{\varepsilon}\right)_{0}+\xi \partial_{x}\left(f_{\varepsilon}\right)_{0}=-\lambda_{\varepsilon}-\partial_{\xi \xi}^{2} \mu_{\varepsilon}
$$

where $\left(\lambda_{\varepsilon}\right)_{\varepsilon>0}$ and $\left(\mu_{\varepsilon}\right)_{\varepsilon>0}$ are nonnegative measures bounded uniformly in $\varepsilon$. Therefore, by Proposition 3.9, $\rho_{\varepsilon}$ and $\rho_{\varepsilon} u_{\varepsilon}$ are locally compact.

Proof. - We set $\lambda_{\varepsilon}=\left(M_{0}\left[f_{\varepsilon}\right]-M_{0}^{*}\left[f_{\varepsilon}\right]\right) / \varepsilon$ and $h_{\varepsilon}=\left(\left(f_{\varepsilon}\right)_{0}-M_{0}\left[f_{\varepsilon}\right]\right) / \varepsilon$. We have $\lambda_{\varepsilon} \geqslant 0$ thanks to Lemma 3.4. Since $\int_{\mathbb{R}} h_{\varepsilon} \mathrm{d} \xi=0, \int_{\mathbb{R}} \xi h_{\varepsilon} \mathrm{d} \xi=0$ and $h_{\varepsilon}$ has compact support in $\xi$, there exists a distribution $\mu_{\varepsilon}$ with compact support in $\xi$ such that $h_{\varepsilon}=$ $\partial_{\xi \xi}^{2} \mu_{\varepsilon}$. Thus we have (3.55). We integrate this equality and get

$$
\iiint_{] 0, T[\times \mathbb{R} \times \mathbb{R}} \lambda_{\varepsilon} \leqslant \iint_{\mathbb{R} \times \mathbb{R}} f_{0}^{0}(x, \xi) \mathrm{d} x \mathrm{~d} \xi .
$$

Take now a test function $\varphi(t, x, \xi)=\varphi_{1}(t, x) \varphi_{2}(\xi)$, with $\varphi_{1}, \varphi_{2}$ nonnegative and of class $C_{c}^{\infty}$, and define $\phi \in C^{\infty}$ by $\varphi_{2}=\partial_{\xi \xi}^{2} \phi$. We have that $\phi$ is convex, and by the entropy 
minimization principle

$$
\left\langle\mu_{\varepsilon}, \varphi\right\rangle=\left\langle\partial_{\xi \xi}^{2} \mu_{\varepsilon}, \varphi_{1} \phi\right\rangle=\iiint_{10, T[\times \mathbb{R} \times \mathbb{R}} \varphi_{1} \phi h_{\varepsilon} \geqslant 0,
$$

thus $\mu_{\varepsilon} \geqslant 0$. We integrate now (3.55) against $\xi^{2} / 2$, and we get

$$
\iiint_{] 0, T[\times \mathbb{R} \times \mathbb{R}} \mu_{\varepsilon} \leqslant \iint_{\mathbb{R} \times \mathbb{R}} \frac{\xi^{2}}{2} f_{0}^{0}(x, \xi) \mathrm{d} x \mathrm{~d} \xi,
$$

which concludes the proof.

Proof of Theorem 3.8 when $\gamma=3$. - The beginning of the proof is the same, we can replace compensated compactness by Proposition 3.10, but it remains to get that $f_{\varepsilon}-M^{*}\left[f_{\varepsilon}\right] \rightarrow 0$ a.e. $t, x, \xi$. For a subsequence, we have

$$
\rho_{\varepsilon} \rightarrow \rho, \quad \rho_{\varepsilon} u_{\varepsilon} \rightarrow \rho u \quad \text { a.e. } t, x,
$$

with $(\rho, u) \in \widetilde{D}$. The bound (3.53) implies that for a subsequence,

$$
\frac{1}{2}\left[\left(\xi-u_{\varepsilon}\right)^{2}-3 \kappa\left(\rho_{\varepsilon}^{*}\right)^{2}\right]\left[\left(f_{\varepsilon}\right)_{0}-M_{0}\left(\rho_{\varepsilon}^{*}, u_{\varepsilon}, \xi\right)\right] \rightarrow 0 \quad \text { a.e. } t, x, \xi .
$$

Since $\rho_{\varepsilon}-\rho_{\varepsilon}^{*} \rightarrow 0$ a.e., it gives

$$
\left[\left(\xi-u_{\varepsilon}\right)^{2}-3 \kappa \rho_{\varepsilon}^{2}\right]\left[\left(f_{\varepsilon}\right)_{0}-M_{0}\left(\rho_{\varepsilon}, u_{\varepsilon}, \xi\right)\right] \rightarrow 0 \quad \text { a.e. } t, x, \xi,
$$

and we recall that this quantity is nonnegative.

We set $E=\{(t, x) \in] 0, T[\times \mathbb{R} ; \rho(t, x)>0\}$. On $E$, we have $u_{\varepsilon} \rightarrow u$ a.e., and from (3.58), $\left(f_{\varepsilon}\right)_{0} \rightarrow M_{0}(\rho, u, \xi)$ a.e. $\xi$ since the set of $\xi$ such that $(\xi-u(t, x))^{2}=3 \kappa \rho^{2}(t, x)$ has measure zero. Then, for any bounded domain $B$ in $(t, x)$, by passing to the limit as $\varepsilon \rightarrow 0$ in

$$
\begin{aligned}
& \iint_{(t, x) \in B \cap E} \int_{\xi}\left(f_{\varepsilon}\right)_{0} \mathrm{~d} t \mathrm{~d} x \mathrm{~d} \xi+\iint_{\substack{(t, x) \in B \\
(t, x) \notin E}} \int_{\xi}\left(f_{\varepsilon}\right)_{0} \mathrm{~d} t \mathrm{~d} x \mathrm{~d} \xi \\
& =\iint_{(t, x) \in B \cap E} \int_{\xi} M_{0}\left[f_{\varepsilon}\right] \mathrm{d} t \mathrm{~d} x \mathrm{~d} \xi+\iint_{\substack{(t, x) \in B \\
(t, x) \notin E}} \int_{\xi} M_{0}\left[f_{\varepsilon}\right] \mathrm{d} t \mathrm{~d} x \mathrm{~d} \xi,
\end{aligned}
$$

we get that

$$
\iint_{\substack{(t, x) \in B \\(t, x) \notin E}} \int_{\xi}\left(f_{\varepsilon}\right)_{0}(t, x, \xi) \mathrm{d} t \mathrm{~d} x \mathrm{~d} \xi \rightarrow \underset{\substack{(t, x) \in B \\(, x) \notin E}}{\int} \int_{\xi} M_{0}(\rho, u, \xi) \mathrm{d} t \mathrm{~d} x \mathrm{~d} \xi=0 .
$$

Finally we get that, up to an extraction,

$$
\left(f_{\varepsilon}\right)_{0} \rightarrow M_{0}(\rho, u, \xi) \quad \text { a.e. } t, x, \xi
$$


Since $\left(f_{\varepsilon}\right)_{1}=\xi\left(f_{\varepsilon}\right)_{0}$, we also get $\left(f_{\varepsilon}\right)_{1} \rightarrow \xi M_{0}(\rho, u, \xi)=M_{1}(\rho, u, \xi)$ a.e., and the result follows.

\section{Pressureless model}

This section is devoted to the proof of Theorems 1.1-1.3 when $p=0$. We build a sticky blocks dynamics with mass loss that solves the system for particular data, that is used to approximate arbitrary initial data.

The analysis is similar to that of [2], and differs from the one for the system of pressureless gases without constraint, that gives Dirac distributions on $\rho$ in finite time (see $[5,9,12])$. The entropies and entropy fluxes are defined by

$$
\eta_{S}(\rho, u)=\rho S(u), \quad G_{S}(\rho, u)=\rho u S(u)
$$

for any $S: \mathbb{R} \rightarrow \mathbb{R}$ convex. They satisfy

$$
\eta_{S}^{\prime}(1, v) \cdot(1, v)=S(v)
$$

thus the entropy inequalities (1.11) write

$$
\partial_{t}(\rho S(u))+\partial_{x}(\rho u S(u)) \leqslant Q S(v) .
$$

\subsection{Sticky blocks dynamics}

Let us consider a volume fraction $\rho(t, x)$ and a momentum density $\rho(t, x) u(t, x)$ given by

$$
\rho(t, x)=\sum_{i=1}^{n} \mathbb{1}_{a_{i}(t)<x<b_{i}(t)}, \quad \rho(t, x) u(t, x)=\sum_{i=1}^{n} u_{i}(t) \mathbb{1}_{a_{i}(t)<x<b_{i}(t)},
$$

with $a_{1}(t)<b_{1}(t) \leqslant a_{2}(t)<b_{2}(t) \leqslant \cdots \leqslant a_{n}(t)<b_{n}(t)$. The time evolution is defined as follows. The number of blocks $n$ indeed depends on $t$, but is piecewise constant. As long as the blocks do not meet, they move at constant velocity $u_{i}(t)$. When two blocks collide at a time $t^{*}$, the dynamics is exhibited in Fig. 1, and is defined as follows. The volume fraction $\rho$ is given locally by

$$
\rho(t, x)= \begin{cases}\mathbb{1}_{a_{l}(t)<x<b_{l}(t)}+\mathbb{1}_{a_{r}(t)<x<b_{r}(t)} & \text { if } t<t^{*}, \\ \mathbb{1}_{a_{l}(t)<x<b_{r}(t)} & \text { if } t^{*} \leqslant t<t_{f}, \\ \mathbb{1}_{a(t)<x<b(t)} & \text { if } t \geqslant t_{f}\end{cases}
$$

and the momentum density $\rho u$ by

$$
\rho u(t, x)= \begin{cases}u_{l} \mathbb{1}_{a_{l}(t)<x<b_{l}(t)}+u_{r} \mathbb{1}_{a_{r}(t)<x<b_{r}(t)} & \text { if } t<t^{*}, \\ u_{l} \mathbb{1}_{a_{l}(t)<x<c(t)}+u_{r} \mathbb{1}_{c(t)<x<b_{r}(t)} & \text { if } t^{*} \leqslant t<t_{f}, \\ u^{*} \mathbb{1}_{a(t)<x<b(t)} & \text { if } t \geqslant t_{f} .\end{cases}
$$




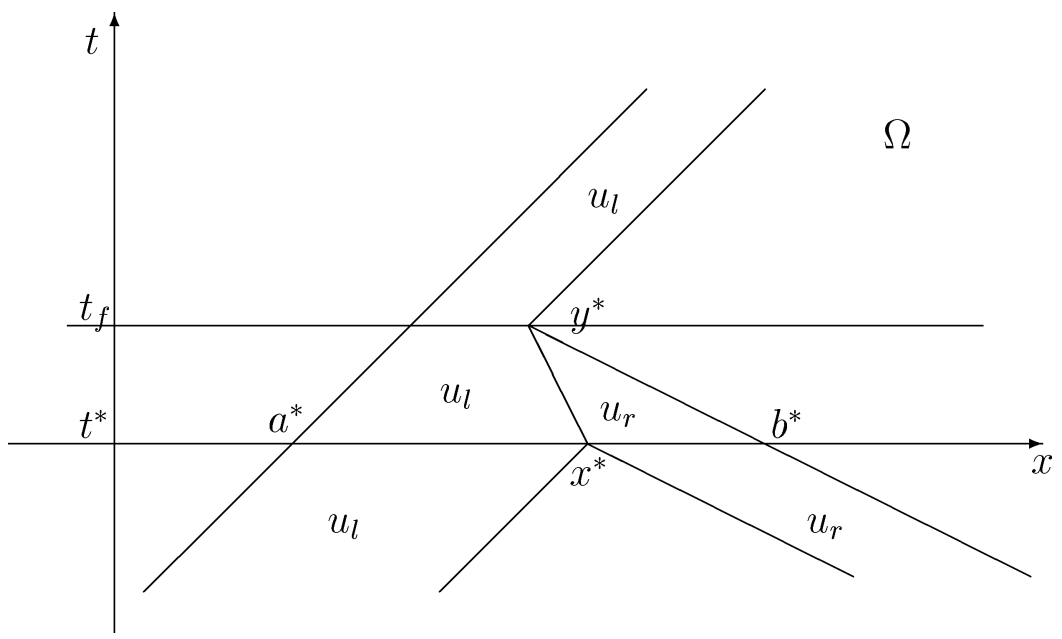

Fig. 1. Collision of two blocks.

We have $a_{l}(t)=a^{*}+u_{l}\left(t-t^{*}\right), b_{l}(t)=x^{*}+u_{l}\left(t-t^{*}\right), a_{r}(t)=x^{*}+u_{r}\left(t-t^{*}\right)$, $b_{r}(t)=b^{*}+u_{r}\left(t-t^{*}\right), c(t)=x^{*}+u_{\mathrm{loss}}\left(t-t^{*}\right), a(t)=a_{l}\left(t_{f}\right)+u^{*}\left(t-t_{f}\right)$ and $b(t)=b_{r}\left(t_{f}\right)+u^{*}\left(t-t_{f}\right)$, with $u_{r} \leqslant u_{\text {loss }} \leqslant u_{l}$,

$$
\begin{gathered}
t_{f}=\min \left(t^{*}+\left(b^{*}-x^{*}\right) /\left(u_{\text {loss }}-u_{r}\right), t^{*}+\left(x^{*}-a^{*}\right) /\left(u_{l}-u_{\text {loss }}\right)\right), \\
u^{*}= \begin{cases}u_{l} & \text { if }\left(x^{*}-a^{*}\right) /\left(u_{l}-u_{\text {loss }}\right)>\left(b^{*}-x^{*}\right) /\left(u_{\text {loss }}-u_{r}\right), \\
u_{r} & \text { if }\left(x^{*}-a^{*}\right) /\left(u_{l}-u_{\text {loss }}\right)<\left(b^{*}-x^{*}\right) /\left(u_{\text {loss }}-u_{r}\right) .\end{cases}
\end{gathered}
$$

One of the right or left block disappears, and in case of equality in (4.8), $a(t)=b(t)$ and all the mass disappears.

When more than two blocks collide at the same time, or if a block collides at a time $t_{1}$ with two blocks that are colliding since a time $t^{*}$ with $t^{*}<t_{1}<t_{f}$, we perform the collisions locally at each interface between two blocks. We notice that with this construction, $\rho$ and $\rho u$ are both continuous in time with values in $L^{1}(\mathbb{R})$.

It only remains to define the interface velocity $u_{\text {loss }}$, and we can indeed take any relation

$$
u_{\mathrm{loss}}=\Phi\left(u_{l}, u_{r}\right)
$$

where $\Phi$ is defined for $u_{l}>u_{r}$ and satisfies

$$
u_{r} \leqslant \Phi\left(u_{l}, u_{r}\right) \leqslant u_{l}
$$

\subsection{Properties of sticky blocks}

We have the following consistency result.

THEOREM 4.1. - There exists a nonpositive measure $Q(t, x) \in \mathcal{M}(] 0, \infty[\times \mathbb{R})$ with concentrations only in $x$, such that with $\rho(t, x)$ and $u(t, x)$ defined by (4.4), and with the 
above defined dynamics, we get a solution to the weak formulation (1.18)-(1.19), (4.3), with $v=u_{l}+u_{r}-u_{\text {loss. }}$.

Proof. - As long as there is no collision, each block moves at the constant velocity $u_{i}$, and $(\rho, u)$ solves the pressureless Euler system. In a neighborhood of initial data, the proof is given in [2]. Let us now look at the case of a collision of two blocks at a time $t^{*}$. Let $\varphi(t, x)$ be a smooth function with support in $\Omega$ of Fig. 1 such that there is no other contact in $\Omega$. We have

$$
\begin{aligned}
& \int_{t^{*}}^{t_{f}} \int_{a_{l}(t)}^{c(t)}\left[S\left(u_{l}\right) \partial_{t} \varphi(t, x)+S\left(u_{l}\right) u_{l} \partial_{x} \varphi(t, x)\right] \mathrm{d} t \mathrm{~d} x \\
& =S\left(u_{l}\right)\left\{\int_{t^{*}}^{t_{f}}\left[\frac{\mathrm{d}}{\mathrm{d} t} \int_{a_{l}(t)}^{c(t)} \varphi(t, x) \mathrm{d} x-u_{\mathrm{loss}} \varphi(t, c(t))+u_{l} \varphi\left(t, a_{l}(t)\right)\right] \mathrm{d} t\right. \\
& \left.\quad+\int_{t^{*}}^{t_{f}} u_{l}\left(\varphi(t, c(t))-\varphi\left(t, a_{l}(t)\right)\right) \mathrm{d} t\right\} \\
& =S\left(u_{l}\right)\left\{\int_{a_{l}\left(t_{f}\right)}^{c\left(t_{f}\right)} \varphi\left(t_{f}, x\right) \mathrm{d} x-\int_{a^{*}}^{x^{*}} \varphi\left(t^{*}, x\right) \mathrm{d} x+\int_{t^{*}}^{t_{f}}\left(u_{l}-u_{\mathrm{loss}}\right) \varphi(t, c(t)) \mathrm{d} t\right\},
\end{aligned}
$$

and similarly

$$
\begin{aligned}
& \int_{t^{*}}^{t_{f}} \int_{c(t)}^{b_{r}(t)}\left[S\left(u_{r}\right) \partial_{t} \varphi(t, x)+S\left(u_{r}\right) u_{r} \partial_{x} \varphi(t, x)\right] \mathrm{d} t \mathrm{~d} x \\
& \quad=S\left(u_{r}\right)\left\{\int_{c\left(t_{f}\right)}^{b_{r}\left(t_{f}\right)} \varphi\left(t_{f}, x\right) \mathrm{d} x-\int_{x^{*}}^{b^{*}} \varphi\left(t^{*}, x\right) \mathrm{d} x+\int_{t^{*}}^{t_{f}}\left(u_{\mathrm{loss}}-u_{r}\right) \varphi(t, c(t)) \mathrm{d} t\right\} .
\end{aligned}
$$

Thus by addition

$$
\begin{aligned}
& \int_{t^{*}}^{t_{f}} \int_{\mathbb{R}}\left[\rho S(u) \partial_{t} \varphi+\rho S(u) u \partial_{x} \varphi\right] \mathrm{d} t \mathrm{~d} x \\
& =\int_{\mathbb{R}}(\rho S(u) \varphi)\left(t_{f}, x\right) \mathrm{d} x-\int_{\mathbb{R}}(\rho S(u) \varphi)\left(t^{*}, x\right) \mathrm{d} x \\
& \quad+\int_{t^{*}}^{t_{f}}\left[\left(u_{l}-u_{\mathrm{loss}}\right) S\left(u_{l}\right)+\left(u_{\mathrm{loss}}-u_{r}\right) S\left(u_{r}\right)\right] \varphi(t, c(t)) \mathrm{d} t
\end{aligned}
$$

and finally

$$
\left\langle\partial_{t}(\rho S(u))+\partial_{x}(\rho u S(u)), \varphi\right\rangle
$$




$$
\begin{aligned}
& =-\left\langle\rho S(u), \partial_{t} \varphi\right\rangle-\left\langle\rho u S(u), \partial_{x} \varphi\right\rangle \\
& =-\int_{t^{*}}^{t_{f}}\left[\left(u_{l}-u_{\text {loss }}\right) S\left(u_{l}\right)+\left(u_{\text {loss }}-u_{r}\right) S\left(u_{r}\right)\right] \varphi(t, c(t)) \mathrm{d} t .
\end{aligned}
$$

Therefore we have (1.31) with

$$
Q_{S}=-\left[\left(u_{l}-u_{\mathrm{loss}}\right) S\left(u_{l}\right)+\left(u_{\mathrm{loss}}-u_{r}\right) S\left(u_{r}\right)\right] \mathbb{1}_{t^{*}<t<t_{f}} \delta(x-c(t)) .
$$

For $S \equiv 1$, we get

$$
Q(t, x)=-\left(u_{l}-u_{r}\right) \mathbb{1}_{t^{*}<t<t_{f}} \delta(x-c(t)),
$$

and for $S=\mathrm{Id}$, we get

$$
(Q v)(t, x)=-\left(u_{l}-u_{r}\right)\left(u_{l}+u_{r}-u_{\mathrm{loss}}\right) \mathbb{1}_{t^{*}<t<t_{f}} \delta(x-c(t)) .
$$

Since $u_{l}>u_{r}$ is necessary to have the collision, we have $Q \leqslant 0$, and (4.13) gives $v=u_{l}+u_{r}-u_{\text {loss. }}$. We prove now the entropy weak product inequality. Since

$$
Q S(v)=-\left(u_{l}-u_{r}\right) S\left(u_{l}+u_{r}-u_{\mathrm{loss}}\right) \mathbb{1}_{t^{*}<t<t_{f}} \delta(x-c(t)),
$$

we obtain, by using that $u_{r} \leqslant u_{\text {loss }} \leqslant u_{l}$, which imply that $u_{r} \leqslant v \leqslant u_{l}$, that for $S$ convex,

$$
\partial_{t}(\rho S(u))+\partial_{x}(\rho u S(u))=Q_{S} \leqslant Q S(v)
$$

which concludes the proof.

Similarly to the model with pressure Lagrange multiplier of [8], we have the discrete Oleinik entropy inequality

$$
u_{i}(t)-u_{i-1}(t) \leqslant \frac{a_{i}(t)-b_{i-1}(t)}{t} \text { for } 2 \leqslant i \leqslant n .
$$

Extending the value of $u(t, x)$ to all $x$ by linear interpolation between two blocks and by putting a constant at infinity, we get that the sticky blocks dynamics satisfies Oleinik's condition (1.26). Then, we observe that according to (4.12)-(4.13), the formulas (1.8) hold, and in particular we have

$$
|Q| \leqslant\left|\partial_{x} u\right|
$$

thus $Q \in L_{\text {loc }}^{\infty}(] 0, \infty\left[, \mathcal{M}_{\text {loc }}(\mathbb{R})\right)$. We also have the maximum principle (1.28) and the entropy equality (1.31) with

$$
Q_{S}(t, x)=\left[\frac{u_{l}-u_{\text {loss }}}{u_{l}-u_{r}} S\left(u_{l}\right)+\frac{u_{\text {loss }}-u_{r}}{u_{l}-u_{r}} S\left(u_{r}\right)\right] Q(t, x) .
$$

Thus, we get (1.32) for every $S$ continuous. 


\subsection{Existence of a solution}

We do not detail the proof of Theorem 1.1, since it is very close to that of [2]. The same argument is used to prove that for any $S$ continuous, $\rho_{n} S\left(u_{n}\right) \rightarrow \rho S(u)$. Only Lemma 2.1 is new and gives directly the entropy weak product inequality.

Remark 4.1. - In Theorem 1.1, we also have the existence of $Q_{S} \in \mathcal{M}([0, \infty[\times \mathbb{R})$ such that (1.31) and (1.32) are satisfied.

The proof of Theorems 1.2 and 1.3 is straightforward. As in [2], we approximate the initial data by blocks. For this data, we build the solution by the above sticky blocks dynamics. Uniform bounds follow from the analysis of Section 4.2. Then, we use the stability to get the solution.

\subsection{Loss of the strong extremality relation}

This section is devoted to a counterexample that shows that in the pressureless case, the extremality relation in the strong sense (1.6) is not included in the entropy weak product inequality (1.11). We construct a sequence of sticky blocks solutions to which we can apply the stability theorem, but for which the limit does not satisfy the strong extremality relation $(1-\rho) Q=0$.

We consider initially two blocks of height $1 / 2$,

$$
\rho^{0}(x)=\frac{1}{2}\left(\mathbb{1}_{0<x<1}+\mathbb{1}_{2<x<3}\right), \quad \rho^{0}(x) u^{0}(x)=\frac{1}{2}\left(\mathbb{1}_{0<x<1}-\mathbb{1}_{2<x<3}\right) .
$$

We use the following approximation of these initial data,

$$
\begin{gathered}
\rho_{n}^{0}(x)=\sum_{k=1}^{n} \mathbb{1}_{\frac{2 k-1}{2 n}<x<\frac{2 k}{2 n}}+\sum_{k=1}^{n} \mathbb{1}_{2+\frac{2(k-1)}{2 n}<x<2+\frac{2 k-1}{2 n},} \\
\rho_{n}^{0}(x) u_{n}^{0}(x)=\sum_{k=1}^{n} \mathbb{1}_{\frac{2 k-1}{2 n}<x<\frac{2 k}{2 n}}-\sum_{k=1}^{n} \mathbb{1}_{2+\frac{2(k-1)}{2 n}<x<2+\frac{2 k-1}{2 n}} .
\end{gathered}
$$

Using the dynamics of Section 4.1 with $\Phi\left(u_{l}, u_{r}\right)=\left(u_{l}+u_{r}\right) / 2$, we get a solution $\left(\rho_{n}, u_{n}, Q_{n}, v_{n}\right)$ to (1.18)-(1.19), (1.10)-(1.11) with regularities (1.15)-(1.17), which is indeed given by

$$
\begin{aligned}
& \rho_{n}=\sum_{k=1}^{n} \mathbb{1}_{t+\frac{2 k-1}{2 n}<x<t+\frac{2 k}{2 n}} \mathbb{1}_{x<\frac{3}{2}}+\sum_{k=1}^{n} \mathbb{1}_{2-t+\frac{2(k-1)}{2 n}<x<2-t+\frac{2 k-1}{2 n} \mathbb{1}_{x>\frac{3}{2}},} \\
& \rho_{n} u_{n}=\sum_{k=1}^{n} \mathbb{1}_{t+\frac{2 k-1}{2 n}<x<t+\frac{2 k}{2 n}} \mathbb{1}_{x<\frac{3}{2}}-\sum_{k=1}^{n} \mathbb{1}_{2-t+\frac{2(k-1)}{2 n}<x<2-t+\frac{2 k-1}{2 n} \mathbb{1}_{x>\frac{3}{2}}},
\end{aligned}
$$

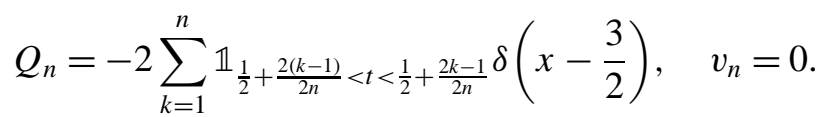

Now, as $n \rightarrow \infty$, one can check easily that

$$
Q_{n} \rightarrow-\mathbb{1}_{\frac{1}{2}<t<\frac{3}{2}} \delta\left(x-\frac{3}{2}\right),
$$




$$
\rho_{n} \rightarrow \rho=\frac{1}{2} \mathbb{1}_{t<x<t+1} \mathbb{1}_{x<\frac{3}{2}}+\frac{1}{2} \mathbb{1}_{2-t<x<3-t} \mathbb{1}_{x>\frac{3}{2}},
$$

and

$$
\rho_{n} u_{n} \rightarrow \rho u=\frac{1}{2} \mathbb{1}_{t<x<t+1} \mathbb{1}_{x<\frac{3}{2}}-\frac{1}{2} \mathbb{1}_{2-t<x<3-t} \mathbb{1}_{x>\frac{3}{2}} .
$$

We use the stability result and we get that $(\rho, u, Q, 0)$ is a solution to (1.18)-(1.19), (1.10)-(1.11) with regularities (1.15)-(1.17) for the initial data $\rho^{0}$ and $\rho^{0} u^{0}$. This solution is half of a usual sticky block solution, as can be easily checked in the pressureless case, we can multiply any weak solution by a factor between 0 and 1 , it is still a solution. It can be also interpreted as the sticky block solution with constraint $\rho \leqslant 1 / 2$. The two blocks with height $1 / 2$ loose their mass when they collide, all the mass disappears though $\rho$ is staying less than $1 / 2$. The extremality relation is lost because here $Q$ does not vanish even if $\rho<1$ everywhere. We get instead $(1 / 2-\rho) Q=0$. However, for the initial data (4.19), we have not been able to find a solution that satisfies the strong extremality relation, and the above weak solution could be considered as the most natural one. One could say that anyway it satisfies the extremality relation if we define $\rho$ to take the value 1 on the line $x=3 / 2,1 / 2<t<3 / 2$. But this indicates clearly that the model introduced here is not satisfactory in the pressureless case. However the phenomenon should not occur with pressure, as indicated by (1.14).

\section{REFERENCES}

[1] L. Barthélemy, Problème d'obstacle pour une équation quasi-linéaire du premier ordre, Ann. Fac. Sci. Toulouse Math. 9 (1988) 137-159.

[2] F. Berthelin, Existence and weak stability for a pressureless model with unilateral constraint, Math. Models Methods Appl. Sci. 12 (2002) 249-272.

[3] F. Berthelin, F. Bouchut, Solution with finite energy to a BGK system relaxing to isentropic gas dynamics, Ann. Fac. Sci. Toulouse 9 (2000) 605-630.

[4] F. Berthelin, F. Bouchut, Kinetic invariant domains and relaxation limit from a BGK model to isentropic gas dynamics, Asymptotic Analysis 31 (2002) 153-176.

[5] F. Bouchut, On zero pressure gas dynamics, in: Advances in Kinetic Theory and Computing, in: Ser. Adv. Math. Appl. Sci., Vol. 22, World Scientific, River Edge, NJ, 1994, pp. 171190.

[6] F. Bouchut, Construction of BGK models with a family of kinetic entropies for a given system of conservation laws, J. Stat. Phys. 95 (1999) 113-170.

[7] F. Bouchut, Renormalized solutions to the Vlasov equation with coefficients of bounded variation, Arch. Ration. Mech. Anal. 157 (2001) 75-90.

[8] F. Bouchut, Y. Brenier, J. Cortes, J.-F. Ripoll, A hierarchy of models for two-phase flows, J. Nonlinear Sci. 10 (2000) 639-660.

[9] F. Bouchut, F. James, Duality solutions for pressureless gases, monotone scalar conservation laws, and uniqueness, Comm. Partial Differential Equations 24 (1999) 2173-2189.

[10] L. Boudin, A solution with bounded expansion rate to the model of viscous pressureless gases, SIAM J. Math. Anal. 32 (2000) 172-193.

[11] Y. Brenier, L. Corrias, A kinetic formulation for multi-branch entropy solutions of scalar conservation laws, Ann. Inst. H. Poincaré Anal. Non Linéaire 15 (1998) 169-190.

[12] Y. Brenier, E. Grenier, Sticky particles and scalar consevation laws, SIAM J. Numer. Anal. 35 (1998) 2317-2328. 
[13] B. Després, Equality or convex inequality constraints and hyperbolic systems of conservation laws with entropy, Preprint, 2001.

[14] R.J. Di Perna, P.-L. Lions, Y. Meyer, $L^{p}$ regularity of velocity averages, Ann. Inst. H. Poincaré Anal. Non Linéaire 8 (1991) 271-287.

[15] W. E, Y.G. Rykov, Y.G. Sinai, Generalized variational principles, global weak solutions and behavior with random initial data for systems of conservation laws arising in adhesion particle dynamics, Comm. Math. Phys. 177 (1996) 349-380.

[16] G. Gagneux, A.M. Lefevère, M. Madaune-Tort, Une approche analytique d'un modèle black-oil des écoulements triphasiques compressibles en ingénierie pétrolière, J. Mécanique Théor. Appl. 6 (1987) 547-569.

[17] E. Grenier, Existence globale pour le système des gaz sans pression, C. R. Acad. Sci. Paris Sér. I Math. 321 (2) (1995) 171-174.

[18] L. Lévi, Problèmes unilatéraux pour des équations non linéaires de convection-réaction, Ann. Fac. Sci. Toulouse Math. 4 (1995) 593-631.

[19] L. Lévi, Obstacle problems for scalar conservation laws, M2AN Math. Model. Numer. Anal. 35 (2001) 575-593.

[20] P.-L. Lions, N. Masmoudi, On a free boundary barotropic model, Ann. Inst. H. Poincaré Anal. Non linéaire 16 (1999) 373-410.

[21] P.-L. Lions, B. Perthame, P.E. Souganidis, Existence and stability of entropy solutions for the hyperbolic systems of isentropic gas dynamics in Eulerian and Lagrangian coordinates, Comm. Pure Appl. Math. 49 (1996) 599-638.

[22] F. Mignot, J.-P. Puel, Inéquations variationnelles et quasivariationnelles hyperboliques du premier ordre, J. Math. Pures Appl. (9) 55 (1976) 353-378.

[23] B. Perthame, E. Tadmor, A kinetic equation with kinetic entropy functions for scalar conservation laws, Comm. Math. Phys. 136 (1991) 501-517.

[24] D. Serre, Relaxation semi-linéaire et cinétique des systèmes de lois de conservation, Ann. Inst. H. Poincaré Anal. Non linéaire 17 (2000) 169-192.

[25] A. Vasseur, Kinetic semidiscretization of scalar conservation laws and convergence by using averaging lemmas, SIAM J. Numer. Anal. 36 (1999) 465-474.

[26] A. Vasseur, Convergence of a semi-discrete kinetic scheme for the system of isentropic gas dynamics with $\gamma=3$, Indiana Univ. Math. J. 48 (1999) 347-364. 